In der Rubrik „Literatur kompakt" werden die wichtigsten Originalarbeiten aus der internationalen Fachliteratur referiert.

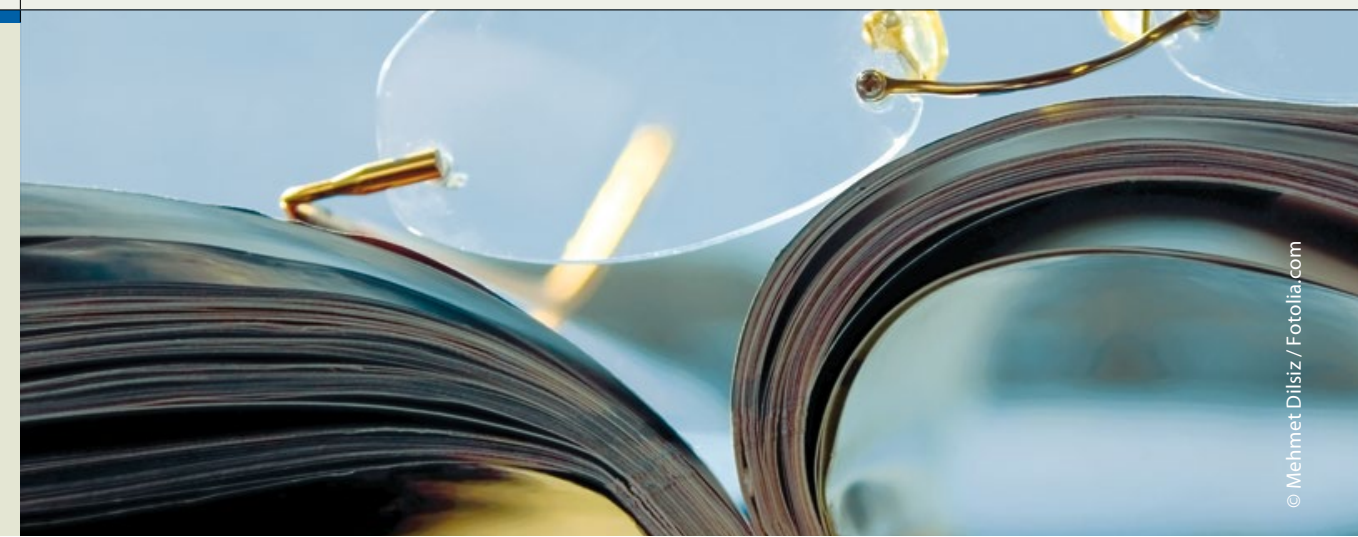

\section{Risikofaktoren für erneutes Wachstum behandelter Hämangiome}

\section{Bei jedem vierten Kind mit einem erfolgreich behandelten Hämangiom kommt es nach Absetzen der Propranololtherapie zu erneutem Wachstum. In einer US-Studie wurde untersucht, wann Rezdive besonders häufig auftreten.}

Propranolol gilt heute als First-LineTherapie zur Behandlung von Hämangiomen bei Kindern. Doch diese Therapieform ist jung und noch bestehen vor allem hinsichtlich Dosierung und $\mathrm{Be}$ handlungsdauer Unsicherheiten. Bei 6-25\% der Patienten kommt es nach Absetzen des Medikaments zu erneutem Wachstum. Im Rahmen einer retrospektiven Kohortenstudie haben Dermatologen aus den USA die Rezidivbildung nach erfolgreicher Propranololtherapie untersucht. Dabei ging es vor allem darum, Risikofaktoren für ein Wiederwachstum von Blutschwämmen zu identifizieren.

Analysiert wurden die Daten von 980 Patienten, die zwischen 2008 und 2013 wegen eines Hämangioms behandelt worden waren. Zu Therapiebeginn waren die Kinder im Mittel 5,6 Monate alt. Die mittlere Maximaldosis Propranolol lag bei $1,95 \mathrm{mg} / \mathrm{kg} / \mathrm{Tag}$, die Behandlungsdauer im Median bei vier Monaten. Der Erfolg wurde anhand von Serienfotos mit einer visuellen Analogskala (VAS) von -100 bis +100 bewertet. $\mathrm{Zu}$ Beginn des Wiederwachstums waren die Kinder im Mittel rund 17 Monate alt. Die Maximaldosis der ersten Therapie wurde bei der durchschnittlich siebenmonatigen Weiterbehandlung beibehalten.

Bei 25,3\% der Patienten begann das Hämangiom erneut zu wachsen. Bei $62 \%$ dieser Fälle entstanden größere Neubildungen, bei denen die Propranololdosis angepasst oder die Behandlung wieder aufgenommen wurde, bei $38 \%$ kleinere, die in der Regel lokal therapiert werden

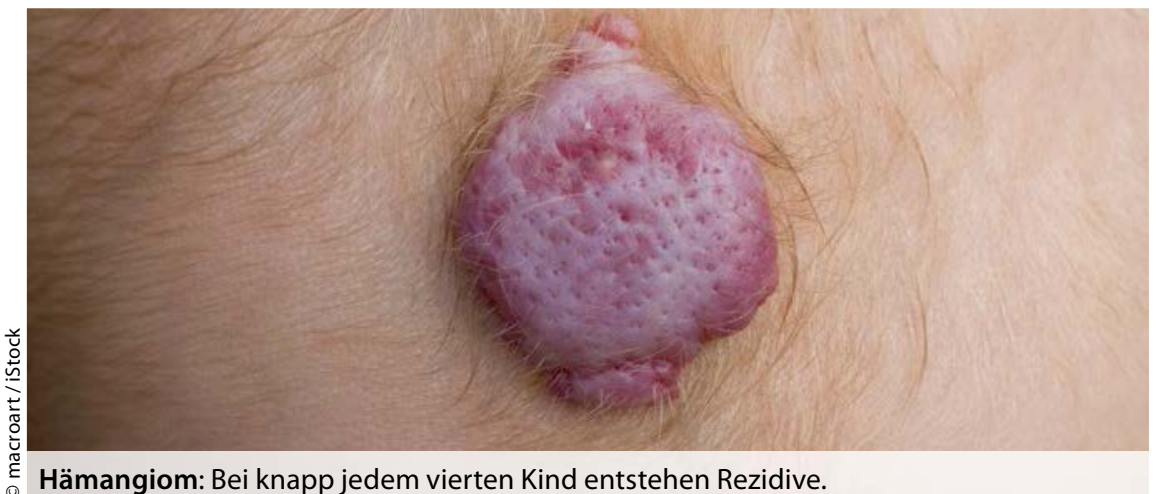

konnten. Bei Kindern, bei denen die Medikation vor Erreichen des neunten Lebensmonats geendet hatte, kam es mehr als doppelt so häufig zu Wiederwachstum als bei Patienten, die bis zum Alter von 12-15 Monaten Propranolol erhalten hatten. Auch das abrupte Absetzen schien sich negativ auf die Rezidivgefahr auszuwirken. Als Risikofaktoren für ein Wiederauftreten wurden in der univariaten Analyse die Aspekte weibliches Geschlecht, Lokalisation im Kopf- oder Halsbereich, segmentale Hämangiome, tiefe und gemischte Hämangiome identifiziert. Wurden in der multivariaten Analyse Faktoren wie Gesamttherapiedauer, Maximaldosis oder Gestationsalter berücksichtigt, waren Mädchen 1,7mal häufiger rezidivgefährdet als Jungen und Patienten mit tiefen Hämangiomen 3,3-mal häufiger als solche mit superfiziellen Hämatomen (Hämangiome mit superfiziellen und tiefen Anteilen: 2,4-mal häufiger als rein superfizielle). Bei $83 \%$ der Kinder mit erneutem Hämangiomwachstum musste das Behandlungsregime angepasst werden.

Fazit: Die Autoren sehen über die Identifizierung der Risikofaktoren die Möglichkeit, diejenigen Kinder im Vorfeld herausfiltern, bei denen zur Vermeidung von Rezidiven einen längere Behandlungsdauer nötig ist. Auch unterstreichen die Ergebnisse die Notwendigkeit individualisierter Therapien, die sich an Indikation, Therapieantwort und den Risikofaktoren für erneutes Wachstum orientieren.

Dr. Christine Starostzik 